\title{
Cada cultura es distinta, cada lengua es distinta... en las personas que hablan las lenguas indígenas está muy arraigado el concepto de comunidad
}

Every culture is different, every language is different... the concept of community is deeply rooted in the people who speak indigenous languages

\section{Esther NAVARRO-HALL}

Traductora e Intérprete ATA

In memoriam

\section{INTRODUCCIÓN}

La profesión de intérprete se caracteriza por la vocación de hacer entender a las partes y contribuir a hacer justicia. Así entendía Esther Navarro-Hall la interpretación y durante sus años de actividad en los tribunales estadounidenses se implicó en cada causa con responsabilidad. Se preocupó por enseñar la interpretación de lenguas indígenas en los EE. UU. y la situación de estas lenguas en México. Como miembro comprometido de la ATA, reivindicó el derecho a expresarse en la lengua vernácula. Esta compañera referente para los que estamos ahora a vueltas con la Interpretación simultánea remota ya realizaba Interpretación a distancia en los años 80 en el Defend Languages Institute.

Palabras clave: interpretación judicial; interpretación en los servicios públicos. 


\section{ENTREVISTA}

Fecha: 11/18/2017

Manuel de la Cruz: Hola, muy buenas tardes. Una vez más, estoy aquí, para presentar a una invitada que viene directamente de los Estados Unidos de Norteamérica. Esther Navarro-Hall, intérprete de conferencias y judicial, y profesora adjunta de Middlebury Institute of International Studies. Ha venido aquí hoy a nuestra Universidad invitada para hablarnos un poco sobre la interpretación judicial y, en especial la experiencia en los Estados Unidos. Estamos muy agradecidos que haya aceptado nuestra invitación hoy para poder hacer esta entrevista. Muy buenas tardes, Esther Navarro-Hall.

Esther Navarro-Hall: Muy buenas tardes, Manuel. Un placer estar con ustedes. Muchísimas gracias por la invitación, estoy encantada de trabajar con ustedes en Salamanca. Nunca había venido a la ciudad, estoy fascinada no sólo por la ciudad sino por el cálido recibimiento que he tenido de ustedes. Muy agradecida, gracias.

MDLC: Muchas gracias. La verdad es que no solamente la interpretación judicial la trae aquí, sino también hemos visto que trabaja muchísimo con el tema de la interpretación remota, uno de los temas que nos gustaría abordar hoy aquí en esta entrevista. En primer lugar, pues ver un poquito la interpretación judicial. ¿Cómo se llega a la interpretación judicial? ¿En Estados Unidos está muy implantado la cuestión de la interpretación? ¿Es como aquí en España que, por un lado, se puede hacer una especie de oposición para ser intérprete en los juzgados y también existen los intérpretes autónomos? ¿Cómo funciona un poquito del tema de la interpretación judicial en los Estados Unidos?

ENH: Sí, mira, Manuel. A grandes rasgos, en Estados Unidos tenemos ya bastantes años de trayectoria en cuanto a la interpretación judicial y jurídica. Hace tiempo se trabajaba más o menos como se hace aquí, pero decidieron por parte del Gobierno estatal y federal que iban a tratar de implementar un sistema en el que se reconocieran los conocimientos de los intérpretes jurídicos y judiciales por medio de una certificación. Tenemos un programa de certificación estatal; hay un consorcio que incluye casi a todos los estados de los Estados Unidos, que participan en este tipo de examen, y una certificación a nivel federal, un examen distinto también. Bastante apreciado porque no muchas personas pasan el examen, entonces está ya a otro nivel. Se llega a la interpretación judicial o jurídica por medio de la formación y mucho trabajo de por medio. Hay algunas personas que estudian una licenciatura específicamente para este tipo de interpretación y otras, la maestría que combina también un poco de interpretación jurídica y judicial. Entonces, se utilizan las mismas modalidades que se usan acá, que son las simultánea, la consecutiva y la traducción a la vista, pero claro la especialidad es distinta, el protocolo es distinto y la ética también tiene ciertos aspectos que hay que tomar en cuenta que son distintos. Se trabaja mucho también con asociaciones en Estados Unidos que ayudan con la diseminación o la difusión de este tipo de trabajo.

Esther NAVARRO-HALL

Cada cultura es distinta, cada lengua es distinta... en las personas que hablan las lenguas indígenas...
CLINA

vol. 7-1, June 2021, 121-128

elSSN: 2444-1961

Ediciones Universidad de Salamanca - CC BY-NC-ND 
MDLC: Existen grandes diferencias probablemente entre Estados Unidos, por ejemplo, y también usted ha hablado hoy también de México, ¿no? En México hay muchas lenguas indígenas que se hablan y ha hablado de un tema que creo que es muy interesante, sobre todo a la hora de garantizar los derechos de un inculpado, de una persona que está acusada en un procedimiento, y el derecho a hablar en su propia lengua para ser reconocido. Me interesaría que nos explica un poco cómo está la situación del tema de las lenguas indígenas a este respecto en México. Sé de buena tinta que usted está muy implicada con estas cuestiones.

ENH: Sí, efectivamente. Estoy trabajando actualmente de forma binacional porque yo voy y vengo entre México y Estados Unidos bastante. Primero como intérprete de conferencias, pero ahora trabajando mucho también con los inicios de la interpretación jurídica y judicial en México. Esta cuestión de las lenguas indígenas es sumamente interesante porque en México se le da el peso que se le da al español. Tenemos 68 lenguas indígenas en México y el español no es el idioma oficial, es el idioma mayoritario, digamos, pero no necesariamente el oficial. Entonces, se le debe dar el mismo peso a todas las lenguas y ahora están empezando a trabajar con la interpretación en lenguas indígenas. Falta mucho por hacer todavía, pero ya y hay muchas personas muy interesadas en que esto se logre bien, sobre todo en cuestión de formación para los intérpretes. Ahora lo que pasa con mucha frecuencia es que, por cuestión de inmigración, muchas personas que hablan lenguas indígenas se vienen a trabajar a Estados Unidos o se van a trabajar a Estados Unidos. Todo lo que implica esto, digamos como la parte social, la parte económica, las personas que necesitan acudir a un hospital por cuestión enfermedad, las personas que tienen algún asunto que arreglar en lo jurídico, lo judicial, no se pueden hacer entender porque no hablan inglés y no hablan español. Entonces, ahí muchas veces interviene el intérprete inglés-español para trabajar en modalidad de relé. Y se necesita información a todo nivel: formación para los jueces, para los abogados, para los intérpretes de inglés-español y para los intérpretes que hablan la lengua indígena. Y sí, como tú decías anteriormente, esto es para hacer valer los derechos de las personas al igual que se deben hacer valer en cualquiera de los otros idiomas. En Estados Unidos decimos que la persona tiene que estar presente para el proceso, presente lingüísticamente y, si no se le proporciona un intérprete para el idioma que él o ella habla, entonces no se considera que está presente en el proceso.

MDLC: También con respecto al tema de los de los derechos, quiero volver otra vez al tema de los indígenas que es una cuestión que creo que es bastante interesante. Sobre todo, porque cuando pensamos en México pensamos solamente en el México que habla español.

ENH: Exacto.

Esther NAVARRO-HALL Cada cultura es distinta, cada lengua es distinta... en las personas que hablan las lenguas indígenas...
CLINA

vol. 7-1, June 2021, 121-128

elSSN: 2444-1961

Ediciones Universidad de Salamanca - CC BY-NC-ND 
MDLC: Y ahora mismo ha quedado patente la cantidad de lenguas que se hablan en México y las diferencias que hay también sociales. Supongo que también tiene que ver. Un acercamiento hacia comunidades, a modos de expresión que, además ha comentado usted una cosa muy interesante en la conferencia, expresan las cosas de otra manera. Me gustaría que nos explicase «de otra manera». ¿Qué diferencias culturales se puede uno encontrar realmente entre una sociedad más, digámoslo así, occidental, como la de México DC, frente a las comunidades indígenas? ¿Qué diferencias culturales podemos encontrar?

ENH: A mí me ha llevado muchos años apenas empezar a entender algunas de las diferencias y es por nuestra forma de pensar que tenemos muy arraigada que tenemos como mexicanos, como mestizos. Cada cultura es distinta, cada lengua es distinta, por supuesto, pero en las personas que hablan las lenguas indígenas está muy arraigado el concepto de comunidad. Esto, por lo general, y no existe tanto en las culturas occidentales. La cosmovisión de ellos o la forma de ver las cosas de ellos, la filosofía de las personas que hablan las lenguas indígenas es muy distinta a la nuestra. A nosotros se nos inculcar desde el principio el guion individualista, la forma de ver la sociedad, la forma de retener propiedad, la forma de manejarnos dentro de nuestra sociedad es muy «por separado». En las comunidades indígenas todo es comunidad, en realidad. Todo es compartido y es muy difícil ver una comunidad de hablantes de lenguas indígenas que se adjudiquen las cosas para sí o que manejen todo de una manera muy individualista. Siempre que vas a algún evento de una de estas comunidades o que vas a trabajar con ellos en alguna cuestión profesional, todo tiene que ver con la comunidad, todos se conocen, comparten muchas cosas. La vida de ellos, la parte cultural desde su nacimiento hasta su muerte, tiene que ver con la comunidad, tiene que ver con la naturaleza, con la madre tierra y están muy apegados a todo lo que es natural. Por lo tanto, es muy difícil y comprender ese estilo de vida porque a nosotros nos han criado de esa manera y tenemos que lograr entender eso, esa parte cultural inclusive, para poder con trabajar de una manera lo más mínima posible, entender de donde provienen ellos y cuál es su sentir y su pensar, para trabajar nosotros como intérpretes de inglés-español con estos colegas de lenguas indígenas. Me parece que un acercamiento, como mencionabas tú anteriormente, es lo ideal. Te voy a dar un ejemplo de lo que mencionaba en la conferencia anteriormente. Nosotros, como intérpretes de inglés-español, tendemos a buscar una equivalencia en el léxico, en la terminología, de igual a igual, por ejemplo, si tú dices la palabra «intérprete» en español, esperas que el inglés sea «interpreter» y viceversa. En las lenguas indígenas, la figura del intérprete no es lo mismo que para nosotros. Ellos tienen que explicar o decir cuál es el concepto de ellos en su lengua. Muchas veces cuando yo he trabajado con colegas que hablan lenguas indígenas y me toca hacer relé con ellos, por curiosidad empiezo a preguntar "Cómo dices tú esto en tu lengua». Entonces, hablando con una colega en el sur de California, estábamos dando un curso, la profesora Holly Mikkelson y yo en Los Ángeles, y era formación para intérpretes de lenguas indígenas. Entonces, pregunté ese

Esther NAVARRO-HALL Cada cultura es distinta, cada lengua es distinta... en las personas que hablan las lenguas indígenas...
CLINA

vol. 7-1, June 2021, 121-128

elSSN: 2444-1961

Ediciones Universidad de Salamanca - CC BY-NC-ND 
día a los alumnos que estaban presentes «¿cómo dices tú intérprete en tu lengua?». Y «¿por qué oigo que cuando dices intérprete en tu lengua se lleva a 6 o 7 palabras para decir lo que yo digo en una?». Es una forma de juzgar de mi parte que no debería hacer, pero me estoy dando cuenta cada vez que trabajo con ellos, que tengo que entender el concepto y le pregunté a esta colega «¿en tu lengua cómo lo dices?». Cada uno tenía una forma distinta: algunos decían «la persona que ayuda», otros «la persona que habla otra lengua", etcétera. Y esta señora me dice: «En mi lengua, intérprete se dice «la persona sagrada que transmite el mensaje» ". Entonces, un concepto más lindo no se me ocurre. Entonces digo "caray, si eso te lleva 7 palabras, se lo puedes decir en 10, es una maravilla». Otra forma de pensar completamente distinta pero muy poética y muy bella.

MDLC: Muy poética, creo que en el fondo es una cosmovisión también muy interesante. Recuerdo que comentó hace un momento en la conferencia el propio concepto de propiedad privada. Por ejemplo, nosotros tenemos un concepto de propiedad privada completamente distinto y el concepto de comunidad que ellos tienen, de compartir.

ENH: Sí. Exactamente mencionaba que el pueblo indígena más conocido de mi estado, del estado de Chihuahua, son los tarahumaras o rarámuris en la lengua de ellos, pero ellos no tienen ese concepto de propiedad privada. Lo que ellos tienen, les pertenece a todos y comparten todo, sea comida, sean implementos para labrar, sea lo que sea. Cuando van a los Estados Unidos, tienen algún problema porque toman algo, llevan algo y, claro, se considera que están robando algo y ellos no alcanzan a entender cómo se pueden robar a sí mismos si lo que tienen les pertenece a todos. Es otro concepto completamente distinto.

MDLC: Aquí, como estamos en Salamanca, en la Escuela de Salamanca, Francisco de Vitoria fue el primero que habló del derecho divino de la propiedad privada y que hemos arrastrado, ha ido a Estados Unidos y ha vuelto.

ENH: Exactamente.

MDLC: Me ha gustado que lo comentara aquí, precisamente en Salamanca. Hablando un poco también de lo importante que es acercar mundo, acercar lenguas y tender puentes entre las culturas, vamos a ver ese otro elemento de nuestro siglo, de esta era de la digitalización que estamos viviendo en estos momentos. Hemos visto que la tecnología nos está ayudando en muchas cosas, que la tecnología por ejemplo puede facilitarnos que todas estas estas lenguas étnicas, estas minorías, puedan comunicarse y tener ese acceso no a la comunicación. Hablando sobre las tecnologías, son muy interesante también los proyectos de los que vas a hablar próximamente en Madrid, que tienes un taller sobre interpretación remota, que parece que es el futuro hacia donde nos dirigimos. Hay algo muy interesante que ha comentado y es que es el momento

Esther NAVARRO-HALL Cada cultura es distinta, cada lengua es distinta... en las personas que hablan las lenguas indígenas...
CLINA

vol. 7-1, June 2021, 121-128

elSSN: 2444-1961

Ediciones Universidad de Salamanca - CC BY-NC-ND 
de definir bien esta cuestión. ¿Podría explicarnos un poquito cómo ve usted el tema de la interpretación remota, hacia dónde nos tendremos que dirigir como profesionales y cómo utilizar esta tecnología?

ENH: Exacto, sí. Es uno de los retos más grandes que tenemos, no sólo en Estados Unidos, sino yo sé que a nivel mundial. La interpretación a distancia o la interpretación remota no es nada nuevo, eso es algo que ya se hacía con distinta tecnología desde hace tiempo. Yo trabajaba en el Instituto de Defensa que se llama Defend Languages Institute en el condado de Monterrey y desde los años 80 recuerdo que a mí me tocaba hacer interpretación a distancia, pero con otra tecnología completamente distinta. Había un desfase entre la persona que decía algo a distancia por vídeo y luego cuando le contestabas, como se veía antes en las noticias que inclusive como que temblaba un poco en la pantalla, el vídeo. Después se entraron otras tecnologías y como mencionaba anteriormente ahora con el WebRTC y otras plataformas se maneja la interpretación a distancia de otra manera. Parece que el único impedimento que existe ahora para hacer interpretación remota en simultánea es el ancho de banda, por lo demás la tecnología ha avanzado mucho, por ejemplo, para hacer interpretación consecutiva hay muchas plataformas, como tú bien sabes, se utilizan ya para otras profesiones. Se trabaja mucho con las conferencias a distancia, por ejemplo, con un vídeo muy nítido y un sonido bastante bueno. Yo mencionaba en una de las conferencias que tuvimos, no sé si fueron en Georgia o en Las Vegas, de una de las asociaciones de intérpretes que, para mí, muchas personas exigen el vídeo en ciertas condiciones, que debe ser este y es el ideal. Pero para mí lo principal es el sonido. Si no puedo oír bien, si no tengo el sonido o la calidad de sonido que debo tener para interpretar, entonces no puedo interpretar. Eso es lo primero. Pero ahora con la tecnología ese obstáculo ya se está sobrepasando. Entonces creo que sí nos incumbe como profesionales lograr que se vayan instituyendo mejores prácticas para trabajar bien con la interpretación a distancia. Como todo, en nuestra profesión es algo que se está viendo como algo nuevo, pero en realidad ya se usa en otras profesiones y como te decía antes, ya se hacía interpretación a distancia desde hace tiempo. Ahora conforme la tecnología se va volviendo más asequible y los intérpretes van logrando, digamos obtener su propia tecnología y su forma de trabajar dependiendo en la localidad, por supuesto, y si tienen wifi, si pueden trabajar en la nube y demás. Esto se va a ser cada vez más común. Hay ventajas y desventajas, por supuesto, de trabajar a distancia. Las ventajas pueden ser que no tienes que viajar tanto, que puedes trabajar cómodamente desde tu hogar o desde alguna oficina, que en ciertas situaciones conviene no estar al lado de la persona a la que vas a interpretar, digamos en el caso de una enfermedad contagiosa o algo así. Y desventajas, falta todavía la parte de la gestión de la interpretación, cómo trabajar, crear algunas pautas que sean más o menos uniformes para todos trabajar de la misma forma, y sobre todo tener ese control de la tecnología para nosotros entenderlo y que al final no nos impongan una forma de trabajar que no sea acordé con nuestras pautas y con nuestra ética profesional.

Esther NAVARRO-HALL

Cada cultura es distinta, cada lengua es distinta... en las personas que hablan las lenguas indígenas...
CLINA

vol. 7-1, June 2021, 121-128

elSSN: 2444-1961

Ediciones Universidad de Salamanca - CC BY-NC-ND 
MDLC: Sí. Yo creo que hay varios problemas que creo que hay que solucionar, también estoy de acuerdo contigo. Uno de ellos también es el tema de los derechos laborales y de las horas en las que cómo trabajo y las condiciones. Tenemos ya la ISO técnica que hemos conseguido establecer, la 2603 de 2016 además, que se hizo la última modificación. Esos son pequeños logros que hemos conseguido. Yo creo que ahora mismo sí que tendríamos que ir por delante, abrir un debate acerca de cómo tienen que cambiar las condiciones de trabajo. Por cierto, y esto es como una anécdota. Sony hace poco presentó una de las películas vía holograma. El actor estaba el actor estaba en Berlín y se hizo una entrevista aquí en un hotel con interpretación simultánea. ¿Crees que podría ser algo interesante? Tener, como usted decía, un holograma e interpretar, poder tener ahí delante a al cliente.

ENH: Mira, viendo hacia el futuro para mí algunas del algunos de los elementos que van a marcar la diferencia en cuestión de tecnología. Yo siempre que converso con los ingenieros y los empresarios del valle de Silicon, porque vivo muy cerca, estamos de acuerdo en que una de las cuestiones que va a cambiar mucho la forma de trabajar con la tecnología es lo que llaman en inglés el input, el ingreso, la forma en que tú te complementas con la máquina, digamos. Hasta ahora todavía es por medio del ratoncito y del teclado... Están haciendo unas cuestiones muy interesantes con guantes que pueden manejar exactamente de manera virtual, digamos. Pero, para mí, hasta que no cambie eso y la máquina o el dispositivo vaya más de acordé al cuerpo humano, creo que falta pegar ese salto todavía. Y, aparte de eso, como mencionabas tú, está la cuestión de los hologramas y la realidad virtual. Hay cosas muy interesantes que están ocurriendo con los juegos, los videojuegos, y parece ciencia ficción, ¿verdad? Efectivamente. Esta cuestión de los implantes y todo esto, pero creo que también posteriormente va a ser algo sí en lo que vamos a tener que fijar nuestra atención. Tarde o temprano, no sé cuántos años haya de por medio, pero tarde o temprano creo que se va a lograr algo muy interesante con esa presencia virtual del intérprete o como dices tú manejando con hologramas y con todo. Ya se ve esa tecnología en el Gobierno, ya se ve en Hollywood... Si estás pensando cómo se va a idear o cómo se va a crear, ya dalo por hecho.

MDLC: Ya se puede pensar. O ya está hecho. Como los coches sin conductor, ya están funcionando, es una cuestión legal. Llevamos ya nuestros 20 minutitos, pero el programa ya se está acabando.

ENH: ¡Qué rápido se pasa!

MDLC: Quiero agradecer muchísimo el que haya aceptado la entrevista. Esther Navarro-Hall, intérprete de ¡conferencias y judicial. Muchísimas gracias por estar aquí. Muchísimas gracias también por dar la conferencia que ha dado tan extraordinaria de nuestros alumnos aquí y a nosotros, a los profesores que hemos aprendido muchísimo. Y nada más, está invitada para cuando quiera volver aquí. Está su casa.

Esther NAVARRO-HALL Cada cultura es distinta, cada lengua es distinta... en las personas que hablan las lenguas indígenas...
CLINA

vol. 7-1, June 2021, 121-128

elSSN: 2444-1961

Ediciones Universidad de Salamanca - CC BY-NC-ND 
ENH: Yo, encantada de la vida. A mí me ha gustado mucho Salamanca y, sobre todo, como te decía, y la fraternidad de los colegas acá y la forma en que me han tratado. Estoy maravillada. Cada vez que pueda, voy a regresar. Te agradezco mucho también lo de la entrevista y acogida. Un placer estar con ustedes.

\author{
Entrevista: «Manuel De la Cruz Recio» \\ Transcripción: Beatriz Guerrero García \\ Enlace: https://programadondelenguas.blogspot.com/2017/12/entrevista-esther-navarro-hall.html \\ Duración: 25:45:00
}

\title{
NOTA BIOGRÁFICA
}

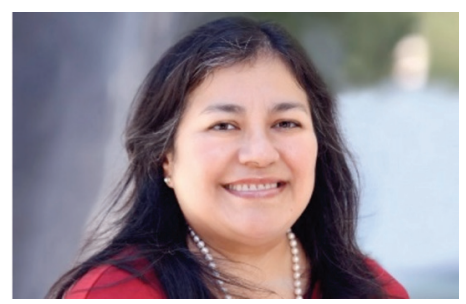

Esther Navarro-Hall, miembro de ATA, fue una Intérprete de conferencias y judicial y profesora adjunta del Middlebury Institute of International Studies. Dedicó parte de su vida profesional al Instituto de Defensa, Defend Languages Institute, en el condado de Monterrey en los años 80, así como al Departamento de Estado de los EE. UU. Es considerada una pionera en el uso de las nuevas tecnologías aplicadas a la interpretación consecutiva-simultánea y desarrolló el método Sim-Consec ${ }^{\mathrm{TM}}$. Destaca su compromiso con los pueblos indígenas en México. En respuesta al terremoto de 2017, organizó la «Interpreter Brigade» para coordinar la ayuda humanitaria y facilitar su distribución en las zonas de habla no-hispana.
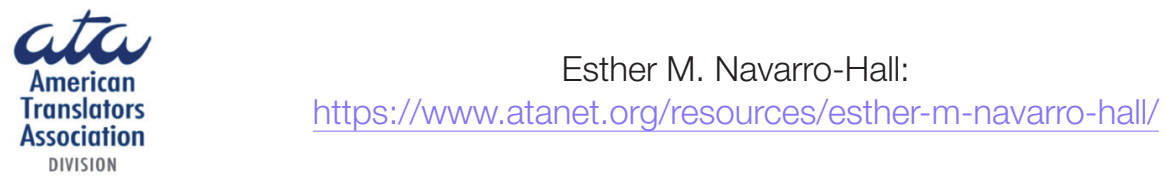

Esther M. Navarro-Hall:

DIVISION

\section{BIBLIOGRAFÍA}

Gudbergsdottir, Eva. 2008. Institute Mourns the Loss of Professor and Alumna Esther Navarro-Hall https://www.middlebury.edu/institute/news/institute-mourns-loss-professor-and-alumna-esther-navarro-hall [Consulta: 5/11/2018]

Esther NAVARRO-HALL

Cada cultura es distinta, cada lengua es distinta... en las personas que hablan las lenguas indígenas... 